\title{
Proyectos de Vida, Género Y MATERNIDAD EN EL PERÚ
}

\author{
Life, gender and maternity projects in Peru
}

\author{
Aymé Buitrón Aranda ${ }^{1}$
}

\begin{abstract}
RESUMEN
Este artículo tiene por objetivo mostrar la valoración de la sociedad respecto a la maternidad, sobre todo desde la perspectiva de las mujeres. Para cumplir este fin hemos hecho una revisión bibliográfica y también nos hemos basado en dos investigaciones que hicimos sobre el tema. Las variables que presentamos para explicar la valoración de la maternidad son las siguientes: aspectos sociales vinculados a la maternidad de la mujer en el campo peruano, los proyectos de vida de las mujeres, la búsqueda de afecto en función de la maternidad, la valoración que tienen ellas del trabajo fuera del hogar y de los estudios universitarios. Nuestro hallazgo ha sido encontrar que existe relación entre el proyecto de vida, los estudios universitarios y la valoración de la maternidad. Las madres adolescentes, por problemas en su autoestima y falta de proyectos alternativos de vida tienen una valoración extrema de la maternidad; en cambio, las jóvenes universitarias valoran la maternidad tanto como los varones con quienes estudian.
\end{abstract}

Palabras claves: maternidad, madre adolescente, género, proyectos de vida.

\begin{abstract}
This article aims to show society's appreciation of motherhood, especially from the perspective of women. To achieve this goal we have done a bibliographic review and we have also based on two researches we did on the subject. The variables we present to explain the assessment of motherhood are as follows: social aspects linked to the motherhood of women in the Peruvian society, the life projects of women, the search for affection according to motherhood, valuation of out-of-home work superior studies. We found that there is a relationship between the life project, university studies and the assessment of motherhood. Adolescent mothers, because of self-esteem problems and lack of alternative life projects, have an extreme appreciation of motherhood; instead, young university girls value motherhood as much as the young men they study with.
\end{abstract}

Keywords: motherhood, teen-age mother, gender, life projects.

1 Profesora asociada. Departamento de Estadística, Demografía, Humanidades y Ciencias Sociales. Facultad de Ciencias y Filosofía, Universidad Peruana Cayetano Heredia

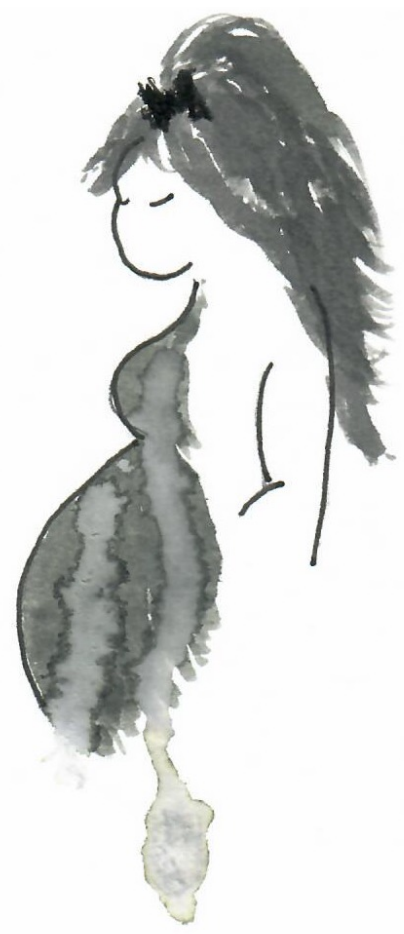

®Aldo Vivar

\section{LA MATERNIDAD COMO PROYECTO DE VIDA}

$\checkmark$

a maternidad no es un hecho natural sino una construcción cultural (Knibiehler, 2001 p.36). En la antigüedad la palabra maternidad no existía ni en griego ni en latín, aunque la función de madre estaba presente en las mitologías. La importancia de la función era la maternal para la renovación de los grupos sociales, de tal manera que para compensar la elevada mortalidad era necesario 
tener muchos hijos. En el siglo XII aparece el término maternitas, acompañado de la palabra paternitas, y de esta manera exaltaron el culto mariano, sin dejar de despreciar la maternidad carnal de Eva. En la época de la Ilustración la maternidad espiritual y la carnal se aproximan y comienza a formularse el modelo de buena madre, siempre sumisa al lado del padre y se le valoriza sólo por la crianza de los hijos y la relación afectiva complementa la función nutricia. La glorificación del amor materno se desarrolla el siglo XIX hasta los años sesenta del siglo XX. (Knibiehler, 2001 pp. 40-41).

La religión en el Perú impuso un código estricto de comportamiento sexual que impuso severas penas a quien se alejara del paradigma de la virgen María. En la versión bíblica la mujer es despojada de su voluntad y debe someterse a la voluntad del hombre y la desobediencia la condena al desamparo (Knibiehler, 2001 pp. 15-21). La mujer que perdía su virginidad era tachada de egoísta, frívola e impura, por lo que debía llegar casta al matrimonio. Los hombres aprendieron que los prostíbulos eran necesarios porque la esposa no podía estar sometida a la rutina sexual de esos lugares, la esposa debía ser tratada con una rutina sexual breve y ascética (Knibiehler, 2001 pp.51-57). El marianismo es un fenómeno latinoamericano en el que se exalta la dignidad del sufrimiento, la castidad, la obediencia y la frigidez post nupcial y es la contraparte del machismo (Barrig, 2017, p.58).

Tradicionalmente, el bienestar de la mujer descansa en la experiencia de ser parte activa de una relación afectiva, esta identidad del yo supone un problema constante diferente a los hombres. Se supone que una mujer puede vivir periodos prolongados o hasta la vida entera sin sexo, pero no puede vivir sin participar activamente en una relación interpersonal con amigos, familia o pareja (Dio Bleichmar, 1991, pp.85-87).
Los estudios sobre la relación temprana madrehijo nos hablan de bebes que desarrollan de manera normal física y psíquicamente, con el cuidado de una buena madre que definen como: disponible, comprensiva, cuidadora y cariñosa, o sea una empresa de servicio unipersonal (Dio Bleichmar, 1991, p.97).

A partir de los años sesenta, la maternidad comienza a abordarse por la demografía, con temas vinculados a la fecundidad y el uso de anticonceptivos; también se estudia desde la antropología, sobre todo las mentalidades a través del estudio de la historia personal, la familia y la infancia de la madre. Algunos estudios abordan el estatus social de la mujer y su maternidad, exponiendo las experiencias de las madres a partir de la técnica de la historia de vida y se reconstruye la experiencia de las mujeres como madres (Buitrón, 2003, p.39).

Desde nuestros inicios profesionales ha sido nuestra preocupación estudiar los estereotipos de la mujer, nuestro interés inicial se vinculaba al abandono infantil y me preguntaba: ¿Qué puede hacer que sus padres los lleven a exponerse a tantos peligros en las calles? ¿Por qué concebirlos si no se les puede proteger en su infancia? Este problema, lamentablemente común en el Perú, mellevó a estudiar su génesis. Es así que llegué al tema de la maternidad y encontré que el tener hijos no es siempre una elección si no el resultado de procesos sociales que generan el abandono infantil.

Entre los procesos sociales que generan el abandono infantil tenemos a la maternidad adolescente y la pobreza de la familia del niño. Si recordamos que el $70 \%$ de los pobres son mujeres (ONU, 2009, p.1), se puede entender más fácilmente que el género, la pobreza y el abandono infantil se pueden vincular. Entonces, debemos comprender porque la mujer cae en dicha situación de pobreza y los 
problemas a los que se vincula frecuentemente dicha situación.

El desempleo y la pobreza están muy relacionados (ONU 2014, p.183). En el 2010, la tasa de participación en la fuerza de trabajo de la mujer continuaba estando por debajo del $30 \%$ en África septentrional y Asia occidental; por debajo del $40 \%$ en Asia meridional; y, por debajo del $50 \%$ en el Caribe y América Central. A pesar de que la participación de la mujer ha aumentadoenlaseconomíasmás desarrolladas, la fuerza de trabajo femenina encuentra empleo predominantemente en los servicios. Este sector representa al menos tres cuartas partes del empleo de las mujeres en la mayoría de las regiones más desarrolladas, en América Latina y el Caribe. La agricultura representa más de la mitad del empleo de las mujeres y los hombres en el África subsahariana (con exclusión de África meridional) y de las mujeres en Asia meridional. El sector servicios y la agricultura ofertan empleos de tipo vulnerable, sobre todo en África, Asia y América y ellas también son trabajadoras por cuenta propia o trabajadoras familiares no remuneradas (ONU, 2014 p.100). Es así que debemos concluir en que la pobreza afecta más a la mujer por la precariedad de su empleo, pero ese no es el único factor que influye en la pobreza de género (ONU, 2014, p. 176).

Sobre la pobreza de género debemos agregar que en algunas partes del mundo, las mujeres y las niñas sufren más los efectos de ella en su hogar; por ejemplo, si en un hogar los padres pobres deben elegir a quien enviar al colegio o la universidad, preferirán enviar al varón por considerar que ellos deben mantener económicamente el hogar y la mujer debe ser mantenida, porque ella es quien debe cuidar a los hijos.
Los datos disponibles muestran que los hogares presididos por mujeres tienen mayor probabilidad de ser pobres que los hogares del mismo tipo cuyo jefe es un varón. Lo que se observa en América Latina y el Caribe y en las regiones más desarrolladas, es que los hogares de madres solteras con hijos tienen tasas de pobreza más elevadas que los de padres solteros con hijos (ONU, 2014 p. 177).

Vemos que en los hogares tradicionales pobres se prepara menos a la mujer para el trabajo fuera de la casa, en lo que corresponde a su instrucción y también vemos que ellas tienen más probabilidades de ser pobres que los varones. Es en ese contexto que debemos entender cómo algunas mujeres pueden verse atrapadas en su maternidad y descuidan a los hijos, exponiéndolos al peligro del trabajo infantil.

En un estudio previo hemos encontrado que la maternidad tradicional dependiente es frecuentemente parte de un contexto de violencia y paternidad irresponsable (Buitrón, 2003, p.84-86), sobre todo en el caso de la maternidad que comienza en la adolescencia. En las líneas siguientes vamos a explicar cómo la pobreza y la violencia se unen en contra del bienestar de los niños y de las mujeres envueltos en esas circunstancias.

Las relaciones de pareja que implican desigualdad de poder y autoritarismo del varón parten de hogares de origen en que se da la desvalorización de la mujer como ser humano independiente, autónomo y se le percibe como incapaz para desarrollarse fuera del hogar.

Es muy importante la socialización en la niñez y sobre todo en los primeros años para forjar personas responsables y equilibradas (Buitrón, 
2003, pp.61-68). Los psicólogos plantean que la base emocional de los primeros años de vida es crucial para el desarrollo del niño porque el amor satisfecho conduce a la autoestima y la generosidad; en cambio, el amor insatisfecho lleva a la desadaptación y el resentimiento (Buitrón, 2003, p.61).

Según diversos estudios, el completo y feliz desarrollo de los niños parece descansar en la experiencia empática con sus padres, en el estudio de Portocarrero vemos la relación que existe entre la formación de una persona violenta y la educación autoritaria y sin diálogo en el hogar, la pobreza en sí no forja personas con baja autoestima, desadaptación y resentimiento, características que llevan a la violencia, pero la falta de diálogo y la violencia psicológica o física sí generan violencia (Portocarrero 1998, p.224-225).

Carmen Pimentel estudia poblaciones de bajos ingresos en el distrito de Comas en Lima (Perú), formados por un $60 \%$ de parejas convivientes, que un $30 \%$ inició su convivencia porque la mujer quedó embarazada entre los 13 y 18 años, el 14\% de mujeres dice que comenzó la convivencia porque buscaba el amparo y protección para ella y sus hijos, y el $6 \%$ de mujeres son de poca edad y se unieron a hombres de 50 a 60 años (Pimentel, 1988 p.55). Observamos que en más del $30 \%$ de sus casos de estudio, la maternidad prematura estuvo muy vinculada al inicio de la convivencia y a la dependencia económica; es decir, existe una relación desigual dentro de la pareja o de desequilibrio de poder. El desequilibrio de poder en la pareja lleva frecuentemente a la violencia, la cual es lamentablemente un problema cada vez más común en nuestra vida cotidiana y vemos que ella se genera en hogares disfuncionales como los que estamos señalando.
En diversos estudios se encuentra que la maternidad adolescente se genera en parte por la falta de diálogo en el hogar y la búsqueda de afecto. De lo que podemos deducir que las madres adolescentes provienen de hogares disfuncionales y este no será un problema fácil de superar en el hogar que formara, problema que se añade al problema de pobreza que generalmente atraviesan.

Vemos que los casos vinculados a la convivencia por embarazo son comunes en el caso de las adolescentes, quienes frecuentemente ven frustradas sus expectativas futuras dentro de un embarazo no planificado.

En adelante pasamos a desarrollar el caso de las madres adolescentes, quienes presentan proyectos de vida muy vinculados a la pobreza y se desarrollan en hogares disfuncionales.

\section{La Maternidad Adolescente}

La maternidad adolescente en el Perú era de un $12 \%$ entre los 15 y 19 años en 1999, pero esta cifra ha aumentado al 14,6\% (INEI, 2015, p. 126). Se observa que el 2009 la tasa de maternidad adolescente se incrementa en 2,7\% a los 15 años y a los 19 años en 31,3\%; además, cerca al 60\% de adolescentes mujeres hubiera querido hacerlo posteriormente, en 1990 sólo el 30\% decía lo mismo. Vemos que a pesar de que ha aumentado el deseo de no embarazarse, el embarazo adolescente no disminuye, las adolescentes que sí deseaban embarazarse han disminuido del $52,6 \%$ al $31,7 \%$. Por esta razón es que debemos comprender que los proyectos de vida de las adolescentes no corresponden a la vida que finalmente tienen, se han dado algunos cambios en la mentalidad, hay un mejor manejo de información sobre la sexualidad pero son otros factores las que las llevan al embarazo no deseado (Mendoza W, Subiría G., 2013, pp. 471-479). 
El embarazo adolescente es del 56,7\% en las adolescentes sin educación, el 34,9\% en las adolescentes coneducación primaria y del11,6\% en adolescentes con educación secundaria y 4,5\% con educación superior. Queda claro que la falta de instrucción se vincula a la realidad de la madre adolescente. Además, entre las adolescentes que se encuentran en el quintil inferior de riqueza la maternidad adolescente es mayor $(24,0 \%)$ y en las del quintil superior de riqueza $(7,4 \%)$ es mucho menor (INEI, 2015, p.129). El embarazo adolescente rural es casi el doble que en el área urbana y en la selva es del 27,5\% para el 2012. La falta de proyectos alternativos de vida en las adolescentes, como la posibilidad de estudiar y de trabajar, motiva a la maternidad temprana, así el grupo de 18 a 19 años, generalmente fuera del sistema educativo, muestra prevalencias superiores al $20 \%$ de embarazo.

En las adolescentes más pobres, el embarazo llevará a conseguir un empleo a edades más tempranas y bajo malas condiciones laborales; $\mathrm{y}$, en tal sentido se perpetúan las inequidades. La permanencia en la escuela secundaria puede modificar las percepciones delas adolescentes respecto de las oportunidades de trabajo que perderían si la dejan, mejoraría su autoestima e iniciativa personal, desincentivándolas del riesgo de un embarazo no planificado. También, esta mayor retención del sistema escolar puede incidir en el uso del tiempo por parte de las adolescentes, dedicándose a sus estudios (Mendoza, 2013, p. 31).

En Colombia, el programa Subsidio Educativo ha contribuido a reducir el embarazo adolescente, siendo la condición no sólo asistir a la escuela secundaria sino también alcanzar un mínimo de rendimiento educativo (Mendoza, 2013, pp.33-34). El Gobierno de México ha incorporado a su oferta de programas sociales, en gestión con los gobiernos locales y organizaciones comunales y Organizaciones
No Gubernamentales, un programa de becas para adolescentes madres y gestantes. En otras regiones del mundo se han registrado logros semejantes (Mendoza, 2013, p.36).

La relación entre maternidad adolescente y la convivencia es muy determinante, ya que la convivencia no se presenta como producto de una libre elección si no como el mejor resultado esperado frente a una maternidad no planificada.

Veíamos al inicio del artículo que las mujeres tienen más probabilidades de ser pobres que los varones, quienes por más que tengan hijos no se hacen responsables de ellos. La mayor educación correlaciona con mejores ingresos económicos y en el caso del embarazo adolescente encontramos que se vincula a la deserción escolar; en conclusión, los niños y jóvenes sin educación adecuada enfrentan un mercado laboral escaso y de muy bajos salarios.

Uno de los hallazgos que hice en una investigación, que realicé en 1999, fue que las madres adolescentes no deseaban enviar a sus hijos a trabajar y ellas querían dedicarse sólo a cuidarlos, pero todo terminó en una realidad muy diferente ya que ellas tenían que salir a trabajar a la calle con sus hijos, el deseo de ser protegidas por un varón dentro de sus hogares no era posible, aunque ellas lo deseasen. El trabajo infantil no es sólo una expresión de la pobreza, si entendemos el trabajo infantil como una desprotección y negligencia en la crianza infantil, la podemos entender como violencia contra el niño. Así Ana Jusid (1991, pp. 30-62), en su estudio para Latinoamérica, nos habla de la situación de conflicto familiar de la madre adolescente, además de su soledad, la ausencia de la figura paterna, la incomunicación, la búsqueda de afecto, los modelos contradictorios de figura materna $\mathrm{y}$ otros problemas. 
Para el caso de Chile, Salazar y Matamala (1994, pp. 157-160) encuentran que las madres adolescentes crecen en un medio de liberalidad sexual pero sin la adecuada comunicación con la madre. La madre de la adolescente generalmente tiene muy poco tiempo libre porque trabaja y afronta muchos problemas por su precariedad económica, por lo que no logra que su hija desarrolle una adecuada valoración e identificación con ella; y, no llegan a establecer lazos de confianza ni adecuada comunicación con sus hijas, exigiendo de ellas básicamente responsabilidad y apoyo en el hogar para la atención de sus hermanos. Las autoras señalan que la falta de autoestima y de espacios para la afectividad familiar de la madre adolescente está vinculada a la desafección al uso de métodos anticonceptivos, ya que el embarazo es el resultado de su búsqueda de atención y afecto.

Un hogar sin diálogo y sin relaciones de afecto promueve que los hijos salgan en busca de alternativas fuera del hogar y las adolescentes se embarazan en esta búsqueda y sus niños se desarrollan sin la protección debida. En el estudio que realicé en 1999 a partir del seguimiento y desarrollo de historias de vida de madres adolescentes captadas en el Instituto Materno Perinatal de Lima, encontramos dos grupos, una de maternidad adolescente voluntaria y otra de maternidad adolescente involuntaria (Buitrón, 2003, p.82). Respecto a su zona de residencia, tanto las madres voluntarias como las madres involuntarias vivían en áreas periféricas de Lima, específicamente en asentamientos humanos de los distritos de Independencia, San Juan de Lurigancho, San Juan de Miraflores, Comas y en zonas antiguas y tugurizadas del Cercado de Lima y del distrito del Rímac. Es evidente la situación de gran precariedad material en la vida cotidiana de las madres adolescentes, así se puede entender las presiones económicas a las que ellas se han visto sometidas al igual que sus familias de origen. Ellas no han salido de esta situación de pobreza, algunas ni siquiera se mudaron de las casas paternas o maternas (9 del total) y las otras salieron a viviendas independientes pero igualmente pobres (5 del total). Respecto a su lugar de nacimiento, ocho nacieron en Lima Metropolitana y seis fuera de la capital. Encontramos que sólo cinco padres nacieron en Lima Metropolitana, los demás nacieron en provincias, principalmente en la sierra del Perú (12 de 28). En este sentido, vemos que muchos rasgos de la cultura andina se encuentran en las madres adolescentes nacidas en Lima. Los estudios señalan que el sentido solidario y colectivista, poco individualista, es propio de la idiosincrasia de las poblaciones de las provincias que están fuera de la capital y de los jóvenes de colegios estatales de la capital. Dichos estudios concluyen que estos valores influyen en la maternidad temprana (León, 1996, pp. 11-44), en tanto la socialización con una alta valoración de las relaciones familiares enfatiza en que la mujer priorice siempre este aspecto en su realización personal, lo que al combinarse con la falta de afecto en el hogar de origen lleva a la búsqueda de afecto fuera del hogar de manera temprana.

En el estudio que realicé en 1999 (Buitrón, 2003, p.106), las adolescentes señalaban sentirse motivadas a ser madres de manera temprana porque deseaban retener a su pareja al lado, piensan que los hijos unen a la pareja. Ellas se consideran completas y realizadas sólo cuando son madres, el logro familiar y afectivo es más valorado que el logro laboral. Ellas consideran que un hombre puede trabajar fuera del hogar porque es fuerte físicamente y así puedehacerse respetar ante otros hombres; por lo tanto, es su obligación mantener económicamente a su pareja e hijos, pero como no es tierno y expresa poco sus sentimientos no puede entenderse 
con los niños. En cambio, la mujer es tierna y cariñosa, se le engríe más porque tiene que ser más dulce y atenta con la familia, debido a que su obligación es atender y servir a todos en su hogar y cuidar a los niños. Ellas consideran que es un logro que sus parejas mantengan el hogar, porque es la obligación del hombre y no de la mujer buscar dicho sustento económico.

La madre reacciona de manera diferenciada ante las conductas de sus hijos varones o mujeres, la imitación y el reforzamiento forman a los niños de acuerdo al sexo de nacimiento. Una niña que tiene una buena percepción de su propio género está motivada para cumplir los roles aprendidos (Hyde, 1995pp.59-62).

Las madres adolescentes del estudio consideraban que las niñas tenían la obligación de estudiar y atender en su hogar desde los diez años o antes y que poco a poco aprendían las tareas del hogar, en cambio los niños sólo debían jugar y estudiar (Buitrón, p.134); aquí debemos agregar que esto coincide con lo que señala la literatura sobre la crianza en los grupos nativos en la Amazonía (Brown, Michael, 1984, pp.54-56).

Las madres adolescentes consideran que si la mujer trabaja fuera del hogar, igualmente tiene que dedicarse a las tareas de su casa y el dinero que la mujer obtiene de su trabajo es para todo el grupo familiar. Las madres adolescentes denominan a sus madres abandonadas por sus parejas, madre-padre, porque consideran que no es función de una mujer el trabajo fuera del hogar, actividad que han realizado sus madres debido precisamente a que fueron abandonadas por sus parejas. El concepto de abandonadas es algo que las madres adolescentes mencionan porque consideran que sus madres no pudieron retener a sus parejas, menoscabando este hecho la estima que sienten por ellas (Buitrón, 2003, p. 135).

\section{El ESTATUS QUE DA LA MATERNIDAD, PROBLEMAS DE AUTOESTIMA Y BÚSQUEDA DE AFECTO}

Las madres adolescentes encuentran que el respeto que ahora los demás tienen por ellas es algo de lo que antes de ser madres no gozaban y que las satisface mucho. El género del hijo es importante para las madres adolescentes, porque si tienen un hijo varón ellas consideran que disfrutarán de la seguridad económica que su hijo les dará. Algunas madres adolescentes mencionan el hecho de que por la belleza de la mujer el varón se sentiría más estimulado a mantenerla, protegerla y a la vez la mujer tendría que subordinarse y aceptarlo como jefe y protector (Buitrón, 2003, pp. 109-110). Aquí debemos agregar que algunos estudios encuentran que existe vergüenza por la fealdad en la mujer y equiparan este sentimiento al sentimiento de la impotencia sexual en el varón (Dio Bleichmar, 1999, p.205), por lo que muchas veces su baja autoestima puede asociarse al sentimiento de fealdad.

Las madres adolescentes encuentran en su entorno social pocas expectativas sobre sus logros académicos o laborales. Dentro de sus hogares sus padres le dieron más relevancia a las funciones de apoyo de la adolescente en el hogar que a las actividades académicas que debía llevar a cabo (Buitrón, 2003, p. 136). Como consecuencia de todo lo anterior, las madres adolescentes no culminan sus estudios en el colegio ni buscan trabajo fuera del hogar, desde el momento en que empiezan a convivir con su pareja. En el estudio que realizamos en 1999 encontré lo siguiente:

Respecto a la relación con sus padres señalaban poca comunicación y falta de apoyo afectivo de ambos respecto a su persona. La relación con el padre es en todos los casos distante y en algunos casos se explicita la presencia de maltrato físico, alcoholismo o drogadicción, siempre asociado 
al incumplimiento de la obligación paterna de llevar el sustento económico al hogar. Con la madre señalaban un trato respetuoso pero distante, además consideraban que su madre no era su amiga. En algunos casos se registró el abandono del hogar por la madre por lo que algunas adolescentes crecieron con otros familiares como abuelos o tíos (Buitrón 2003, p. 85).

En resumen, puedo decir que las madres adolescentes establecen una relación de subordinación con sus parejas porque la joven tiene pocas alternativas de vida y grandes necesidades afectivas, las madres involuntarias son quienes se cuidan menos con métodos de planificación familiar, lo cual se vincula al hecho de que fueron a convivir con su pareja a raíz de su embarazo y sienten que exponiéndose a nuevos embarazos legitiman su relación de convivencia. Las madres adolescentes no asumen de manera autónoma su sexualidad ni su maternidad, tendiendo a responsabilizar de esto a su pareja porque ellas consideran que el hecho de que los varones mantengan a los hijos, los hace acreedores al dominio o mando en la relación. Así, la mujer debe subordinarse a él, porque depende económica y afectivamente de su pareja y la madre adolescente asume su tarea reproductiva como central en su vida (Buitrón 2003, p.63).

Vemos que esta realidad se encuentra lejos de ser la ideal para el desarrollo de un niño, ya que los padres no son realmente conscientes de la responsabilidad afectiva y social que tienen entre sus manos. Lo ideal sería un horizonte donde la maternidad no sea un medio de sobrevivencia o una manera de llenar vacíos afectivos o aumentar la autoestima, sino que la maternidad sea una forma de hacer feliz a la niñez, donde cada hombre y mujer entiendan que todos somos realmente responsables de los niños que nacen cada día.
En adelante debemos ampliar la comprensión de la problemática de la maternidad, para lo cual revisaremos el tema de la maternidad entre las mujeres que trabajan y tienen mayor grado de instrucción en el Perú.

\section{La Maternidad, La Educación Y El Empleo}

A partir de 1870 se comienza a dar una educación adecuada a las mujeres de clase media y alta y algunas se desarrollan en la educación superior y profesional. En la segunda mitad del siglo XX, las mujeres obtuvieron igualdad de derechos ante la ley, ingresaron masivamente a la educación escolar y superior y luego muchas pudieron controlar su fertilidad gracias a modernos anticonceptivos (Fuller, 2004 pp. 196-198).

En el Perú, durante el siglo veinte, se ha desarrollado una alta confianza en los estudios en institutos y universidades para el ascenso social, aunque dichos centros de estudios sean poco calificados académicamente (Barrig, 2017, p.11). Los costos de la educación particular, limitan esta educación a la clase media y son mujeres de esa clase social las que llegan primero a la universidad.

El trabajo asalariado en la mujer, a comienzos del siglo veinte en el Perú, no era algo anhelado en la clase media o alta. Se veía mal al hombre que necesitaba que su mujer trabaje, se le veía como incapaz, incluso hasta entrada la década de 1960 las aspiraciones laborales de las mujeres languidecieron con el matrimonio y la maternidad, siempre la mujer necesitaba el permiso de su pareja y apelaba al bienestar del grupo y no como parte de su desarrollo individual ((Barrig, 2017, p. 26-33).

Incluso, en la actualidad, en algunos sectores tradicionales se considera que el trabajo fuera 
del hogar no es necesario para la mujer; y, en los sectores populares del Perú, el matrimonio legal es visto como una alternativa que libra a las mujeres del desamparo. (Barrig 2017, p.56).

La oferta de trabajo para las mujeres no es amplia sobre todo en los sectores populares. En 1972 en Lima Metropolitana el 23,8\% de las mujeres trabajadoras era de empleadas domésticas, frente al 1,4\% de hombres en la misma condición (Del Valle, 1976, p.64). El sector servicios siempre ha captado a mucha mano de obra femenina y entre las mujeres con educación se consideraba que el trabajo de educadora o enfermera era el indicado.

“Entre 1963 y 1973 la matrícula de la población escolar femenina creció a un ritmo del $8 \%$ superior a la masculina que creció al 6,9\%" (Barrig, 2017, p. 261). En la actualidad, el número de mujeres universitarias es superior al número de varones universitarios, incluso esto es una tendencia mundial.

En 1996, el gobierno de Fujimori liberalizó el mercado educativo y dio la bienvenida a la inversión privada y al lucro. En el 2010, la universidad con mayor número de estudiantes fue la Universidad Alas Peruanas con 57616 alumnos, más del doble de los que tenía la UNMSM. El mayor número de alumnos en las universidades peruanas las tienen las universidades privadas como la César Vallejo (37 163 estudiantes), San Martín de Porres, la Católica de los Ángeles de Chimbote, la Andina Néstor Cáceres Velásquez, llevándole mucha distancia a la Pontificia Universidad Católica del Perú con 17531 alumnos (Barrig, 2017, p.111).

Hasta 1960 sólo 4 de cada 100 mujeres jóvenes del campo iban a la escuela; y, se ha dado un cambio importante desde 1961 de tal manera que en el 2007, por primera vez en la historia peruana, la proporción de mujeres rurales jóvenes con educación secundaria o superior fue mayor que la proporción de ellas sin ningún nivel educativo (Boyd, 2013, p.22). Además, en el 2015 se encuentra que tres de cada cuatro estudiantes universitarios son de primera generación (Cuenca, 2015 p.110).

En algunos estudios se encuentra que las mujeres de los sectores medio de Lima, Iquitos y Tarapoto ven la maternidad como la vía más efectiva para que ellas tengan acceso al estatus de adultas y prestigio social, pero no podemos negar que el trabajo ha cobrado enorme importancia en la percepción de sí mismas. Muchas mujeres jóvenes están invirtiendo más tiempo en sus proyectos laborales; pero, entre las jóvenes de menores recursos económicos, las bajas expectativas de encontrar trabajo adecuado las conducen más rápidamente a la maternidad (Fuller, 2004, pp. 204-205).

El discurso actual predica la presencia del padre en la crianza del hijo pero las exigencias laborales no permiten disponer verdaderamente de tiempo para esa actividad (Fuller, 2004 p.207). Se espera de la mujer un menor aporte económico y un mayor aporte en el trabajo doméstico, incluso una de las motivaciones para unirse o casarse, de parte de los varones, es contar con los servicios domésticos de la mujer (Fuller, 2001, pp. 210211).

En un estudio hecho para Ecuador se muestra cómo la migración ha contribuido a la economía de la mujer, lo que es una estrategia de supervivencia de las familias y son las mujeres actoras económicas importantes por sus aportes a la economía del hogar. Las mujeres cuyas identidades de género han cambiado como producto de la experiencia migratoria tienden a posponer su decisión de retorno a su 
lugar de origen, además la modernidad de los medios de comunicación les permiten incluso tomar decisiones en su hogar estando muy lejos ((Herrera, 2004, pp. 390-397).

\section{La Mujer en El Campo Peruano}

Sobre el tema de la mujer de sectores populares y el trabajo en el Perú, Patricia Ruiz-Bravo en su estudio sobre Puno y Lambayeque encuentra en la cultura andina, a diferencia del mundo criollo, que el trabajo es altamente valorado. El que la mujer se defina como trabajadora implica que ella tiene un lugar muy importante en su sociedad. Las mujeres de Puno son socializadas en el trabajo y desde niñas se dedican a muchas actividades cuyo fin es la búsqueda del progreso económico. En cambio, en Talambo, que está en Lambayeque, en la costa peruana, la identidad femenina pasa por el cuidado de los otros, y este sistema patriarcal es la base de su organización social (Ruiz-Bravo, 2004, pp.311-312).

En el caso de Puno, Ruiz-Bravo encuentra que su incorporación al circuito exportador de lana significó una actividad económica vinculada a la hacienda, donde la mujer debía cumplir turnos en la casa del patrón, realizando tareas domésticas, y el varón haciendo el trabajo servil en el campo. Lamentablemente, algunas veces las mujeres eran entregadas por sus parientes al hacendado para evitar represalias y cobros por pérdidas de ganado, considerándose el cuerpo de la mujer como un objeto.

El trabajo en los Andes es un eje importante en las representaciones colectivas, es el medio de obtener posesiones y la laboriosidad es una virtud. Los que más se dedican al comercio son las mujeres, ellas son buenas negociantes y guardianas del dinero; además, cuando se pregunta a las mujeres sí desean participar en el gobierno municipal, la mayoría responde que sí (Ruiz-Bravo, 2004, pp. 286-295).

En el caso de la hacienda Talambo, se desarrollan pequeños propietarios, quienes se vieron obligados a ser asalariados de las haciendas entre 1860 y 1904 y los trabajadores productivos eran los varones. En la actualidad, el papel de proveedor del varón está en crisis y el historial laboral de las mujeres de Talambo es inestable, por lo que las mujeres menores de 30 años son poco entusiastas. Varias mujeres de Talambo han culminado sus estudios de secretarias y oficinistas, pero encontrar trabajo es muy difícil y se sienten frustradas. No es raro que se refugien en una pareja esperando protección y apoyo económico, como hicieron sus madres. Ellas valoran mucho la virginidad, el pudor y el honor sexual, por lo que hay un estigma sobre las mujeres que son abandonadas por sus parejas. Ellas consideran que deben mantener unida a la familia, no se concibe que ella pueda hacerse cargo sola de los hijos, por lo que siempre debe tener una pareja. Además la poligamia masculina se justifica por una supuesta sexualidad natural irrefrenable en los varones. En cambio, la infidelidad femenina es inaceptable y los varones convencen a las mujeres de que la calle es peligrosa por los robos, violaciones y otros, colocando a las mujeres en una situación de temor (RuizBravo, 2004 pp.297-309).

El hecho de que la mujer andina se sienta dignificada con su trabajo no modifica su situación de desigualdad económica frente a los varones. Existen matices importantes entre ambos grupos de mujeres, de Talambo y de Puno, que debemos considerar porque existe una diferencia psicológica entre el tipo de mujer que se considera más autónoma y la mujer psicológica y económicamente dependiente del varón. 
Los estereotipos tradicionales plantean una mujer altamente dependiente de su familia y de las relaciones afectivas y es de esta manera que, tradicionalmente, se educa a la mujer en muchos sectores sociales en el mundo y en el Perú. Cuando hablamos de estereotipos tradicionales no nos referimos exactamente a la cultura andina tradicional de la sierra peruana, porque la mujer campesina de dicho sector acostumbra el trabajo cotidiano diario en el campo y valora su trabajo, el cual considera como indispensable para toda persona y la ociosidad es descalificada.

Ubicando a la mujer en el contexto mundial encontramos que, aunque la mujer trabaje, este esfuerzo no la saca de su pobreza, de tal manera que las mujeres tienen más probabilidad de ser pobres que los hombres cuando viven en hogares unipersonales en muchos países de las regionestantodesarrolladascomoen desarrollo. Las mujeres están sobrerrepresentadas entre los pobres de edad más avanzada en las regiones más desarrolladas; además, en las sociedades más tradicionales del mundo, el derecho y las normas no escritas existentes limitan el acceso de la mujer a la tierra y a otros tipos de propiedad en la mayoría de los países de África y en, aproximadamente, la mitad de los países de Asia. En las regiones menos desarrolladas, son menos las mujeres con ingresos monetarios que los hombres y una proporción significativa de las mujeres casadas no intervienen ni en las decisiones sobre la manera de gastar sus ingresos, sobre todo en los países africanos y en los hogares más pobres (ONU, 2010, p.171). Las mujeres de los quintiles más pobres participan menos en las decisiones del hogar sobre las compras para atender las necesidades cotidianas y son menos las mujeres que los hombres las que tienen ingresos monetarios o poseen tierras $\mathrm{u}$ otros bienes. Este menor acceso a los recursos aumenta la dependencia económica de la mujer con respecto al hombre y la hace más vulnerable a diversos problemas económicos y ambientales (ONU, 2010, pp.187-188).

\section{La Mujer Universitaria}

Podríamos pensar que la educación universitaria es una salida siempre exitosa al proyecto de vida de las mujeres, realmente esto se da solo en parte porque hay varias limitantes propias de la cultura maternal de nuestra sociedad contemporánea.

En el caso que estudiamos, de los jóvenes universitarios de la Universidad Peruana Cayetano Heredia de Lima en Perú, presentaremos algunas características de su personalidad y de su proyecto de vida, haciendo énfasis en la perspectiva de género y de la maternidad. La técnica que utilizamos fue la encuesta a una población de jóvenes estudiantes de cuarto año de estudios de las facultades de la Universidad Peruana Cayetano Heredia. La población de la que partió la encuesta era de 450 alumnos del cuarto año matriculados el 2016 y se determinó un tamaño de muestra con un nivel de confianza del $95 \%$, error absoluto de estimación de 0.05 , asumiendo una proporción de estudiantes con una valoración de tener un hijo o de trabajar en algo relacionado con su profesión de 0.80 ; $\mathrm{y}$, a partir de la fórmula de tamaño de muestra aleatoria se obtuvo un mínimo de 246.

Entre mujeres y varones encontramos las siguientes diferencias (Buitrón, Carbajal 2017, p.21): ellas le dan más importancia a tener amigos, mayor satisfacción con su familia de origen, con los estudios que realiza y con la religión que tienen, mayor aprobación al matrimonio homosexual, menor apertura al riesgo y a arreglárselas solas, así como mayor aprobación a ser obedientes. Ellas manifiestan tener un mayor grado de felicidad y le dan 
mayor importancia a tener una pareja en sus vidas y a formar una familia, así como tener una buena posición económica.

Las mujeres declaran en menor grado que los varones que: están de acuerdo con tener un trabajo relacionado con sus estudios profesionales y desarrollarse como personas, así como en mantener el orden social, lo cual podría expresar su descontento con los roles tradicionales de género, porque a pesar de mostrarse más apegadas que los varones a las relaciones afectivas y familiares, tanto hombres como mujeres declaran por igual el grado de importancia que le dan a tener hijos. Esto evidencia que para ellas todo lo anterior es igual de importante, rompiendo con la alta valoración que las mujeres le dan a la maternidad tradicionalmente.

Además tanto varones como mujeres están de acuerdo con el aborto y el uso de anticonceptivos, ambos no apoyan las aventuras sexuales fuera de la pareja ni el uso de drogas y admiten el divorcio en alguna ocasión (Buitrón, Carbajal, p.32).

Tanto varones como mujeres consideran que es importante: hacer cosas placenteras y consentirse a sí mismos, tomar sus propias decisiones, mostrar lo capaz que son y conservar las costumbres aprendidas (Buitrón, Carbajal, p.33).

En conclusión, las mujeres sí muestran apertura al trabajo fuera del hogar, desarrollo personal y les interesa tener hijos tanto como a los varones y no más que ellos. En cambio, en los sectores populares o madres adolescentes, ellas no muestran apertura al trabajo fuera del hogar y su desarrollo personal está muy vinculado a la maternidad.
En el caso de las estudiantes de enfermería del cuarto año de estudios de la Universidad Peruana Cayetano Heredia vemos diferencias respecto al resto del alumnado de esta universidad. Las estudiantes de enfermería en mención se consideran de clase media y en segundo lugar de clase media baja, lo que contrasta con la mayoría de estudiantes de dicha universidad, quienes se ubican en la clase media o clase media alta. Las estudiantes de enfermería encuestadas del cuarto año de estudios viven en barrios o distritos de los niveles económicos a los cuáles ellas dicen pertenecer como Carabayllo, Independencia, La Victoria, Puente Piedra, San Juan de Lurigancho, San Juan de Miraflores, San Martin de Porres o Villa María del Triunfo. Ellas quieren un trabajo interesante aunque no sea en la profesión elegida, esto se puede vincular a su origen de clase social pero también debemos recordar que en el Perú existe un contexto laboral difícil para la realización profesional.

Las estudiantes de enfermería están en desacuerdo con la importancia que tiene el mandar y decir a los demás lo que tienen que hacer, pero existen estudiantes de otras facultades de la universidad en estudio que no piensan igual y que sí le dan importancia a esta actitud. El mandar y decir a los demás lo que tienen que hacer se vincula al valor que se da al autoengrandecimiento, lo que nos indica que las estudiantes de enfermería no le dan importancia a este tipo de afirmación individual (Buitrón, Carbajal, p.38).

Aquí debemos añadir que el nivel del embarazo en adolescentes peruanas que estudian educación superior ha aumentado durante el 2000 en comparación a los años 90 del siglo 20 (Mendoza, 2013). Esto lo podemos vincular a lo que nos dice Dio Bleichmar (Dio Bleichmar, 1991, p.182) sobre el avance de la mujer 
profesional en su independencia económica y psicológica pero que muchas veces no logra deshacerse de conflictos maternales, ya que ella encuentra que las mujeres son más pegadas al orden y la tradición. Al respecto, agregamos que las estudiantes de enfermería estudiadas consideran que es muy importante ser obedientes y también le dan más importancia al orden que los demás estudiantes, en la medida de que no tienen los mismos recursos materiales para obtener los logros profesionales necesarios para su independencia psicológica y económica. Entendemos su perspectiva tradicional y la mayor importancia que le dan al ser madres y esposas, en comparación a los otros estudiantes de la investigación que realizamos (Buitrón, Carbajal, p. 43).

Tradicionalmente, las mujeres adolescentes no perciben el campo laboral fuera del hogar como relevante, inclusive estudian en la universidad pero no están decididas a hacer una carrera porque para muchas de ellas, el papel de esposa es el más importante y consideran no trabajar en la época de crianza de sus niños, lo que no se puede hacer si desarrollas una carrera profesional. Incluso, la mujer es muy flexible y se prepara para su pareja del futuro y los hijos que tendría (Hyde, 1995, pp.156-157). Cuanto mayor sea el número de hijos que tenga una mujer, menos probable será que trabaje fuera de casa (Nieva y Gutek 1981, p.196). Respecto al bienestar psicológico, los estudios indican que cuanto mayor sea el número de papeles desempeñados, mayor será el estrés, sobre todo si la mujer no encuentra instituciones adecuadas para la atención del niño. Un estudio de 1970 encuentra que la mujer que trabajaba fuera del hogar y dentro del hogar, en total trabajaba 15 horas semanales más que los hombres, considerando el trabajo del hogar ((Nieva y Gutek 1981, pp. 209-212).

Kanter en 1977 demuestra en su investigación que se prefiere a los hombres para puestos más importantes para aquellos que tienen clientes hombres o porque ese es el estereotipo. Se prefiere a las mujeres para puestos de menor importancia, cuando sus clientes son mujeres y cuando las mujeres ocupan puestos directivos no suele reconocerse que reúnan las características adecuadas para ser líderes en forma satisfactoria, (como se cita en Hyde, 1995, p. 203), porque el liderazgo se asocia a la capacidad que se cree tiene la persona para lograr aumentos de sueldos para los subordinados, influencia en las decisiones de los superiores, posibilidad de obtener ascensos y otros.

Instone y colaboradores en 1983 demuestran que cuanto más sea la experiencia en tareas de supervisión que se brinde a las mujeres, mayor será la confianza en su liderazgo (como se cita en Hyde, 1995, pp. 204-205). Mc Mahan, en 1971, estudia la ansiedad de la mujer respecto al éxito o miedo al éxito, pero también debemos resaltar el aporte de Crandrall, en 1969, sobre los descubrimientos vinculados a las diferencias de género en relación a las expectativas de éxito, ya que durante la socialización se tiende a educar de manera diferenciada a varones y mujeres, de tal manera que las mujeres desarrollan menos expectativas que los varones, pero al entrar a una competencia las expectativas de éxito entre los géneros se uniformiza (como se cita en Hyde, 1995, p. 192-193).

Dio Bleichmar encuentra varias causas de la depresión vinculadas a los roles considerados femeninos, los que pasamos a mencionar: la crisis de la edad media por haber perdido su individualidad en mujeres entre 35 y 50 años; las mujeres de edad con el síndrome del nido vacío; las mujeres tradicionales, quienes evitan situaciones que las intimidan; la mujer moderna que trabaja fuera del hogar y se siente culpable 
por no cuidar de sus hijos; las mujeres sin hijos habiendo deseado tenerlos; las mujeres con relaciones adictivas o relaciones desiguales, quienes justifican el sufrimiento constante en la relación de pareja; $y$, las mujeres con culpa porque no llenan las altas exigencias de sus padres respecto al cuidado de los demás (Dio Bleichmar, 1991, pp.124-241).

Vemos así que el no reconocimiento del trabajo que las mujeres desarrollan fuera de su hogar y la culpa que ellas mismas sienten por no dar el cuidado a los hijos o el miedo al éxito laboral, llevan a la depresión y situaciones emocionales de conflicto personal. También, la mujer tradicional, temerosa del mundo fuera del hogar se hace insegura y con baja autoestima, peor aún las mujeres que han internalizado el sufrimiento, la obediencia y la subordinación a la pareja como parte de sus relaciones cotidianas. Siendo así la maternidad un motivo de conflicto no sólo para los estratos populares o sin estudios.

Entender la condición de madre y trabajadora lleva a relacionarse con diversas expresiones de la problemática (Dio Bleichmar, 1991 p.67). Lluís Flaquer trata de comprender la forma de ser madre y profesionales exitosas de un grupo de mujeres en España, a través de un estudio empírico basado en la aplicación del grupo focal como técnica de recojo de información, él entiende que el sentido fundamental de la pareja ya no es la procreación sino la relación afectiva entre dos adultos en situación de plena igualdad que, voluntariamente, se plantean un proyecto de vida en común (Flaquer, 1995, p. 299). La maternidad deja de ser el único elemento central del proyecto vital y de las trayectorias biográficas de las mujeres y pasa a ser un complemento de su profesión, una profesión que en algunos casos se coloca en primera posición en su escala de valores (Flaquer 1995, p. 303).
Las nuevas generaciones de mujeres jóvenes no quieren seguir el mismo modelo de las super mujeres, lo que constituye un factor más a tener en cuenta a la hora de explicar la baja natalidad en los países del sur de Europa. Si se pregunta a las mujeres jóvenes cuáles son sus planes de vida, la mayor parte sigue afirmando que quiere tener hijos; pero, no son pocas las que, no renunciando al deseo de ser madres, posponen esta decisión al máximo con el fin de evitar los cambios negativos que esto puede comportar en sus vidas. Es solo cuando llegan a la edad límite, que a menudo surge un deseo muy fuerte que les plantea una gran urgencia y es entonces cuando el proyecto de la maternidad se intenta llevar a la práctica. También intervienen factores de carácter más estructural, como por ejemplo las dificultades de acceso a la vivienda, la precariedad de la ocupación, la carencia de servicios para las familias que faciliten la conciliación entre la vida familiar y laboral (Flaquer 1995, pp. 298302).

En definitiva, si bien las mujeres han avanzado mucho en la igualdad en relación a los hombres en lo que se refiere a su incorporación en la vida pública, este cambio no se ha visto acompañado de la penetración del hombre en el ámbito del hogar. El exceso de trabajo no sólo empeora su vivencia de la maternidad sino que también hipoteca su vida profesional. Tener que optar por priorizar una u otra dimensión resulta algo necesario para estas mujeres, a diferencia de lo que pasa en el caso de los hombres que no tienen que escoger. El modelo de super mujer implica un estrés y un nivel de presión que las mujeres entrevistadas consideran intolerable. Mientras la vivencia de la paternidad es perfectamente compatible con las aspiraciones profesionales de los hombres, no lo es para ellas. Aquí radica la fuente del conflicto. Por eso ellas reclaman un reparto equitativo de las responsabilidades entre hombres y mujeres, de 
tal manera que se reconozca el derecho de las mujeres a decidir qué tipo de maternidad y qué tipo de trayectoria profesional desean seguir y con qué intensidad. No todos los obstáculos son externos, la propia mujer constituye un impedimento en sí misma, la socialización diferencial de género de estas madres se ve impregnada por el modelo tradicional. Este hecho explica buena parte de la ambivalencia entre la esfera pública y la privada en la mujer, por el hecho de no poder proporcionar el cuidado a sus hijos, lo cual no exigen o no esperan de los hombres. (Flaquer 1995, pp.304305).

\section{REFERENCIAS BibliogRÁFicAS}

1. Barrig, Marruja. Cinturón de castidad- la mujer de clase media en el Perú. Lima: Instituto de Estudios Peruanos; 2017.

2. Brown, Michael F. Una paz incierta. Historia y cultura de las comunidades aguarunas frente al impacto de la carretera marginal. Lima: Centro Amazónico de Antropología y aplicación Práctica; 1984.

3. Boyd, Chris. La transformación de las mujeres jóvenes rurales en el Perú. Análisis a partir de los censos nacionales (19612007). Documento de Trabajo del Programa Nuevas Trenzas, n. ${ }^{\circ}$ 10. Lima: Instituto de Estudios Peruanos; 2013.

4. Buitrón Aranda, Aymé. Violencia, socialización y familiaProblemática de la mujer y el niño. Revista Hontanar Lima: Universidad Peruana Cayetano Heredia. 1999; 3(1): 61-68.

5. Buitrón Aranda, Aymé. Identidad y Maternidad: estereotipos de género, maternidad adolescente y barreras ante la planificación familiar. Primera Edición. Lima: Asociación Peruana de Salud Mental para la Mujer; 2003.

6. Buitron, Aymé y Carbajal, Luz. Informe de Investigación: Auto percepción social, valores y estilo de vida de los estudiantes de la Universidad Peruana Cayetano Heredia, de acuerdo al género. Documento de Trabajo. Lima; 2017.

7. Del Valle, Delma. Factores determinantes de la participación de la mujer en el mercado de trabajo. Documento del Ministerio de Trabajo. Lima: Mimeo; 1976.

8. Dio Bleichmar, Emilce. La depresión en la mujer. Madrid: Temas de Hoy; 1991.

9. Flaquer, Lluís. El modelo de familia española en el contexto europeo. Sarasa, Sebastiá y Moreno, Luis (compiladores). El estado de bienestar en la Europa del sur. Madrid: Consejo Superior de Investigaciones Científicas, Instituto de Estudios Sociales Avanzados; 1995:289-311.

10. Fuller, Norma (2001). Masculinidades. Cambios y permanencias. Varones de Cusco, Iquitos y Lima. Lima: Fondo Editorial de la Pontificia Universidad Católica del Perú.

11. Fuller, Norma. Identidades en tránsito: femineidad y masculinidad en el Perú actual; 2004.
12. Fuller, Norma (editora). Jerarquías en jaque. Estudios de género en el área andina. Lima: Red para el Desarrollo de las Ciencias Sociales en el Perú. CLACSO; 2004: 189-220.

13. Gélis, J. Árbre et le fruit. La naissance dans l'Occident moderne. París: Fayard; 1984.

14. Herrera Gioconda. Género, familia y migración en el Ecuador: lo viejo y lo nuevo. En: Fuller, Norma (editora). Jerarquías en jaque. Estudios de género en el área andina. Lima: Red para el Desarrollo de las Ciencias Sociales en el Perú. CLACSO; 2004:383-406

15. Hyde, Janet Shibley. Psicología de la mujer, la otra mitad de la experiencia humana. España: Morata; 1995.

16. Instituto Nacional de Estadística e Informática. Encuesta Demográfica y de Salud Familiar 2014. Perú: INEI; 2015.

17. Jusid, Ana. Las niñas mamás. Buenos Aires: Sudamericana;1991. Knibiehler Y. La construction sociale de la maternité. Maternité, affaire privée, affaire publique. París: Bayard; 2001.

18. León, Federico. La orientación valorativa del adolescente peruano: hallazgos e implicancias. Morante, Francisco y Soberón, Luis (editores). "Género, sexualidad y población" (pp.). Lima: FOMCIENCIAS; 1996:11-44.

19. Matamala, María Isabel y Salazar, Gloria. Afectividad, género y maternidad en adolescentes. Primer Congreso Nacionalmujer y salud mental. Chile: SERNAM. 1994: 157-160.

20. Mendoza W, Subiría G. El embarazo adolescente en el Perú: situación actual e implicancias para las políticas públicas. Revista Peruana de Medicina y Salud Pública. 2013;30 (3):471-9. URL disponible en: http://www.scielo.org.pe/pdf/rins/ v30n3/a17v30n3.pdf

21. ONU. La mujer en el mundo 2010. Departamento de Asuntos Económicos y Sociales.

22. Nueva York: Publicación de las Naciones Unidas, ST/ESA/ STAT/SER.K/19. 2014. URL disponible en: https://unstats. un.org/unsd/publication/Seriesk/SeriesK_19s.pdf

23. ONU. Género y cambio climático. Ficha informática sobre género y desarrollo \#7. México: Grupo Interagencial de Género del Sistema de las Naciones Unidas de México; 2009. URL disponible en: http:/ / americalatinagenera.org/newsite/ images / cdr-documents / Experiencias/ambiente-1.pdf

24. Pimentel, Carmen. Familia, violencia en la barriada. Lima: TIPACOM; 1998.

25. Portocarrero, Gonzálo. Razones de Sangre. Lima: Fondo Editorial de la Pontifica Universidad Católica del Perú; 1998.

26. Ruiz-Bravo, Patricia. Andinas y criollas: identidades femeninas en el medio rural peruano. En: Fuller, Norma (ed.). Jerarquías en jaque- Estudios de género en el área andina. Lima: Red para el Desarrollo de las Ciencias Sociales en el Perú; 2004: 283-320.

27. Solé, Carlota y Parella, Sónia. Nuevas expresiones de la maternidad. Las madres con carreras profesionales exitosas. Revista de Sociología (Barcelona). 2004; 4, 67-92. URL disponible en: http: / / www.fes-sociologia.com/ files/res / 4/03.pdf

\section{CORRESPONDENCIA:}

ayme.buitron@upch.pe

Fecha de recepción: 04-09-2019.

Fecha de aceptación: 20-12-2019. 